\title{
On Nash-Equilibria of Approximation-Stable Games ${ }^{\star}$
}

\author{
Pranjal Awasthi ${ }^{1}$, Maria-Florina Balcan ${ }^{2}$, Avrim Blum ${ }^{1}$, Or Sheffet ${ }^{1}$, and Santosh \\ Vempala $^{2}$ \\ ${ }^{1}$ Carnegie Mellon University, Pittsburgh, Pennsylvania \\ 2 Georgia Institute of Technology, Atlanta, Georgia
}

\begin{abstract}
One reason for wanting to compute an (approximate) Nash equilibrium of a game is to predict how players will play. However, if the game has multiple equilibria that are far apart, or $\epsilon$-equilibria that are far in variation distance from the true Nash equilibrium strategies, then this prediction may not be possible even in principle. Motivated by this consideration, in this paper we define the notion of games that are approximation stable, meaning that all $\epsilon$ approximate equilibria are contained inside a small ball of radius $\Delta$ around a true equilibrium, and investigate a number of their properties. Many natural small games such as matching pennies and rock-paper-scissors are indeed approximation stable. We show furthermore there exist 2-player $n$-by- $n$ approximationstable games in which the Nash equilibrium and all approximate equilibria have support $\Omega(\log n)$. On the other hand, we show all $(\epsilon, \Delta)$ approximation-stable games must have an $\epsilon$-equilibrium of support $O\left(\frac{\Delta^{2-o(1)}}{\epsilon^{2}} \log n\right)$, yielding an immediate $n^{O\left(\frac{\Delta^{2-o(1)}}{\epsilon^{2}} \log n\right)}$-time algorithm, improving over the bound of [11] for games satisfying this condition. We in addition give a polynomial-time algorithm for the case that $\Delta$ and $\epsilon$ are sufficiently close together. We also consider an inverse property, namely that all non-approximate equilibria are far from some true equilibrium, and give an efficient algorithm for games satisfying that condition.
\end{abstract}

\section{Introduction}

One reason for wanting to compute a Nash equilibrium or approximate equilibrium of a game is to predict how players will play. However, if the game has multiple equilibria that are far apart, or $\epsilon$-equilibria that are far from the true Nash equilibrium strategies, then this prediction may not be possible even in principle. Motivated by this consideration, in this paper we define the notion of games that are $(\epsilon, \Delta)$-approximation stable, meaning that all $\epsilon$-approximate equilibria are contained inside a small ball of radius $\Delta$ (in variation distance) around a true equilibrium, and investigate a number of their properties. If a game is approximation-stable for small $\Delta$, then this means that even if players are only approximately best-responding, or even if the game matrix is not a perfect description of players' true payoffs, stationary play should in principle be predictable. Many natural small 2-player games such as matching pennies and rock-paper-scissors are indeed approximation-stable for $\Delta$ close to $\epsilon$. In this paper we analyze fundamental properties of approximation-stable games.

We show first that all $(\epsilon, \Delta)$ approximation-stable games must have an $\epsilon$-equilibrium of support at most $O\left(\frac{\Delta^{2} \log (1+1 / \Delta)}{\epsilon^{2}} \log n\right)$, yielding an immediate $n^{O\left(\frac{\Delta^{2} \log (1+1 / \Delta)}{\epsilon^{2}} \log n\right)}$. time algorithm for finding an $\epsilon$-equilibrium, improving by a factor $O\left(\Delta^{2} \log (1+1 / \Delta)\right)$

\footnotetext{
* This work was supported in part by NSF grants CCF-0830540 and CCF-0953192, ONR grant N00014-09-1-0751, and AFOSR grant FA9550-09-1-0538.
} 
in the exponent over the bound of [11] for games satisfying this condition (and reducing to the bound of [11] in the worst case when $\Delta=1$ ). Note that by assumption, this approximate equilibrium is also $\Delta$-close to a true Nash equilibrium. We in addition give improved bounds yielding polynomial-time algorithms for the case that $\Delta$ and $\epsilon$ are sufficiently close together. Specifically, for $\Delta \leq 2 \epsilon-6 \epsilon^{2}$ we give an algorithm for finding $O(\epsilon)$-equilibria in time $n^{O(1 / \epsilon)}$. On the other hand, we show that for $\Delta=O(\sqrt{\epsilon})$, there exist $n$-action approximation-stable games in which the Nash equilibrium and all approximate equilibria have support $\Omega(\log n)$, extending results of Feder et al. [10]. We also consider an inverse property, namely that all non-approximate equilibria are far from some true equilibrium, and give an efficient algorithm for finding approximate equilibria in games satisfying that condition.

Note that the classic notion of a stable Nash equilibrium is substantially more restrictive than the condition we consider here: it requires that (1) any infinitesimal deviation from the equilibrium by any player should make the deviating player strictly worse off (a strict equilibrium, implying that the equilibrium must be in pure strategies) and (2) such a deviation should not give the other player any incentive to deviate. Our condition can be viewed in a sense as a weaker, approximation version of requirement (1), namely any deviation by distance $\Delta$ from the equilibrium should make at least one of the two players have at least $\epsilon$ incentive to deviate.

Related Work: There has been substantial work exploring the computation of Nash equilibria in 2-player $n \times n$ general-sum games. Unfortunately, the complexity results in this area have been almost uniformly negative. A series of papers has shown that it is PPAD complete to compute Nash equilibria, even in 2 player games, even when payoffs are restricted to lie in $\{0,1\}[7,1,6]$.

A structural result of Lipton et al. [11] shows that there always exist $\epsilon$-approximate equilibria with support over at most $O\left((\log n) / \epsilon^{2}\right)$ strategies: this gives an immediate $n^{O\left(\log n / \epsilon^{2}\right)}$-time algorithm for computing $\epsilon$-approximate equilibria and has also been shown to be essentially tight [10]. There has also been a series of results $[8,12,5]$ on polynomial-time algorithms for computing approximate equilibria for larger values of $\epsilon$. The best polynomial-time approximation guarantee known is 0.3393 [12].

For special classes of games, better results are known. For example, Barany et al. considered two player games with randomly chosen payoff matrices, and showed that with high probability, such games have Nash equilibria with small support [4].

Our work is also motivated by that of Balcan et al. [2] who consider clustering problems under approximation stability - meaning that all near-optimal solutions to the objective function should be close in the space of solutions - and give efficient algorithms for stable instances for several common objectives. Results relating incentive to deviate and distance to equilibria in general games appear in [9].

\section{Definitions and Preliminaries}

We consider 2-player $n$-action general-sum games. Let $R$ denote the payoff matrix to the row player and $C$ denote the payoff matrix of the column player. We assume all payoffs are scaled to the range $[0,1]$. We say that a pair of mixed strategies $(p, q)$ is an $\epsilon$-equilibrium if for all rows $i$, we have $e_{i}^{T} R q \leq p^{T} R q+\epsilon$, and for all columns $j$, we have $p^{T} C e_{j} \leq p^{T} C q+\epsilon$. We will typically use $\left(p^{*}, q^{*}\right)$ to denote a Nash equilibrium, 
which is an $\epsilon$-equilibrium for $\epsilon=0$. Note that in a Nash equilibrium $\left(p^{*}, q^{*}\right)$, all rows $i$ in the support of $p^{*}$ satisfy $e_{i}^{T} R q^{*}=p^{*} R q^{*}$ and similarly all columns $j$ in the support of $q^{*}$ satisfy $p^{* T} C e_{j}=p^{* T} C q^{*}$.

We also are interested in the distance between mixed strategies. For probability distributions in this context, the most natural notion is variation distance, which we use here. Specifically we define:

$$
d\left(q, q^{\prime}\right)=\frac{1}{2} \sum_{i}\left|q_{i}-q_{i}^{\prime}\right|=\sum_{i} \max \left(q_{i}-q_{i}^{\prime}, 0\right) .
$$

We then define the distance between two strategy pairs as the maximum of the rowplayer's and column-player's distances, that is:

$$
d\left((p, q),\left(p^{\prime}, q^{\prime}\right)\right)=\max \left[d\left(p, p^{\prime}\right), d\left(q, q^{\prime}\right)\right] .
$$

We now present our main definition, namely that of a game being approximation stable.

Definition 1. A game satisfies $(\epsilon, \Delta)$-approximation stability if there exists a Nash equilibrium $\left(p^{*}, q^{*}\right)$ such that any $(p, q)$ that is an $\epsilon$-equilibrium is $\Delta$-close to $\left(p^{*}, q^{*}\right)$, i.e. $d\left((p, q),\left(p^{*}, q^{*}\right)\right) \leq \Delta$.

So, fixing $\epsilon$, a smaller $\Delta$ means a stronger condition and a larger $\Delta$ means a weaker condition. Every game is $(\epsilon, 1)$-approximation stable, and as $\Delta$ gets smaller, we might expect for the game to exhibit more useful structure. Many natural games such as matching pennies and rock-paper-scissors satisfy $(\epsilon, \Delta)$-approximation stability for $\Delta=O(\epsilon)$; see Section 2.2 for analysis of a few simple examples. We note that this definition is very similar to a condition used in Balcan et al. [2] in the context of clustering problems.

All our results also apply to a weaker notion of approximation stability that allows for multiple equilibria, so long as moving distance $\Delta$ from any equilibrium produces a solution in which at least one player has $\epsilon$ incentive to deviate. Specifically,

Definition 2. A game satisfies $(\epsilon, \Delta)$-weak approximation stability if, for any Nash equilibrium $\left(p^{*}, q^{*}\right)$ and any $(p, q)$ such that $d\left((p, q),\left(p^{*}, q^{*}\right)\right)=\Delta,(p, q)$ is not an $\epsilon^{\prime}$-equilibrium for any $\epsilon^{\prime}<\epsilon$.

Organization of this paper: We now begin with a few useful facts about the region around Nash equilibria and the relation between $\epsilon$ and $\Delta$ in any game, as well as a few simple examples of games satisfying $(\epsilon, \Delta)$-approximation stability for $\Delta \approx$ $\epsilon$. We then in Section 3 analyze properties of approximation-stable games, showing that every $(\epsilon, \Delta)$-approximation stable game must have an $\epsilon$-equilibrium of support $O\left(\frac{\Delta^{2} \log (1+1 / \Delta) \log (n)}{\epsilon^{2}}\right)$, yielding an immediate $n^{O\left(\frac{\Delta^{2} \log (1+1 / \Delta) \log (n)}{\epsilon^{2}}\right)}$-time algorithm.

Note that for large $\Delta$ this exponent simply reduces to the $O\left(\frac{\log (n)}{\epsilon^{2}}\right)$ bound of [11], but improves as $\Delta$ approaches $\epsilon$. In Section 5 we give a near-matching lower bound, showing that there exist approximation-stable games with all approximate equilibria having support $\Omega(\log n)$. In Section 4 we analyze games where $\Delta$ is especially close to $\epsilon$, and give polynomial-time algorithms for finding approximate equilibria when $\Delta \leq 2 \epsilon-O\left(\epsilon^{2}\right)$. Finally, in Section 6 we consider the inverse condition that all strategies within distance $\Delta$ of some Nash equilibrium are $\epsilon$-equilibria, and give an efficient algorithm for computing $(\epsilon / \Delta)$-approximate equilibria in this case. 


\subsection{Preliminaries}

We begin with a few preliminary facts that apply to any 2-player general-sum game.

Claim 1. If $(p, q)$ is $\alpha$-close to a Nash equilibrium $\left(p^{*}, q^{*}\right)$ (i.e., if $d\left((p, q),\left(p^{*}, q^{*}\right)\right) \leq$ $\alpha)$, then $(p, q)$ is a $3 \alpha$-Nash equilibrium.

Proof. (omitted)

Claim 1 is useful because while it may be hard to determine how close some pair $(p, q)$ is to a true equilibrium, it is easy to check how much incentive players have to deviate. Say that a Nash equilibrium $\left(p^{*}, q^{*}\right)$ is non-trivial if at least one of $p^{*}$ or $q^{*}$ does not have full support over all the rows or columns. Notice trivial Nash equilibria, if they exist, can be computed in polynomial-time using Linear programming. We then have:

Claim 2. For any nontrivial Nash equilibrium $\left(p^{*}, q^{*}\right)$ and any $\alpha>0$, there exists $(p, q)$ such that $d\left((p, q),\left(p^{*}, q^{*}\right)\right) \geq \alpha$ and $(p, q)$ is an $\alpha$-approximate equilibrium.

Proof. Without loss of generality, assume that $p^{*}$ does not have full support. Let $e_{i}$ be a row not in the support of $p^{*}$. Consider a pair of distributions $\left(p, q^{*}\right)$ where $p=$ $(1-\alpha) p^{*}+\alpha e_{i}$. Since $i$ was not in the support of $p^{*},\left(p, q^{*}\right)$ has variation distance $\alpha$ from $\left(p^{*}, q^{*}\right)$. Yet, in $\left(p, q^{*}\right)$, with probability $(1-\alpha)$ both the players are playing best responses to each other. Hence, no player has more than $\alpha$ incentive to deviate.

Corollary 1. Assume that the game $\mathcal{G}$ satisfies $(\epsilon, \Delta)$-approximation stability and has a non-trivial Nash equilibrium. Then we must have $\Delta \geq \epsilon$.

\subsection{Some Simple Examples}

A number of natural small games satisfy $(\epsilon, \Delta)$-approximation stability for every $\epsilon>0$ and for $\Delta=O(\epsilon)$. Here, we give a few simple examples.

Game 1: The row and the column matrices are $2 \times 2$ as follows:

$$
R=\left[\begin{array}{ll}
1 & 1 \\
0 & 0
\end{array}\right] \quad C=\left[\begin{array}{ll}
1 & 0 \\
1 & 0
\end{array}\right]
$$

Here, the only Nash equilibrium $\left(p^{*}, q^{*}\right)$ is for the row player to play row 1 and the column player to play column 1 , which are dominant strategies. Any deviation by distance $\Delta$ from $p^{*}$ will give the row player $\Delta$ incentive to deviate, regardless of the strategy of the column player. Similarly, any deviation of $\Delta$ from $q^{*}$ will give the column player a $\Delta$ incentive to deviate regardless of the strategy of the row player. Hence, for every $\epsilon \in[0,1]$, this game is $(\epsilon, \Delta)$-stable for $\Delta=\epsilon$.

Game 2: This game is simply matching pennies:

$$
R=\left[\begin{array}{ll}
1 & 0 \\
0 & 1
\end{array}\right] \quad C=\left[\begin{array}{ll}
0 & 1 \\
1 & 0
\end{array}\right]
$$

Denoting the indicator vectors as $e_{1}$ and $e_{2}$, the Nash equilibrium $\left(p^{*}, q^{*}\right)$ is equal to $\left(\frac{1}{2}\left(e_{1}+e_{2}\right), \frac{1}{2}\left(e_{1}+e_{2}\right)\right)$. We now show that for any strategy which is $\Delta$ far from $\left(p^{*}, q^{*}\right)$, at least one player must have $\epsilon$ incentive to deviate for $\epsilon=\Delta \frac{(1+2 \Delta)}{(1+4 \Delta)}$. 
Specifically, let $(p, q)$ be $\Delta$-far from $\left(p^{*}, q^{*}\right)$, and without loss of generality assume $d\left(p, p^{*}\right)=\Delta$. We may further assume without loss of generality (by symmetry) that $p=\left(\frac{1}{2}+\Delta\right) e_{1}+\left(\frac{1}{2}-\Delta\right) e_{2}$. Let $q=\left(\frac{1}{2}-\Delta^{\prime}\right) e_{1}+\left(\frac{1}{2}+\Delta^{\prime}\right) e_{2}$ for $\Delta^{\prime} \in[-\Delta, \Delta]$. In this case the row player is getting a payoff $p^{T} R q=\left(\frac{1}{2}-2 \Delta \Delta^{\prime}\right)$. Furthermore, he can move to row 2 and get payoff $e_{2}^{T} R q=\left(\frac{1}{2}+\Delta^{\prime}\right)$. Hence, the incentive to deviate $\left(e_{2}-\right.$ $p)^{T} R q \geq \Delta^{\prime}(1+2 \Delta)$. Similarly, the column player has payoff $p^{T} C q=\left(\frac{1}{2}+2 \Delta \Delta^{\prime}\right)$, whereas $p^{T} C e_{2}=\left(\frac{1}{2}+\Delta\right)$, and hence has at least $\Delta\left(1-2 \Delta^{\prime}\right)$ incentive to deviate. The maximum of these two is at least $\Delta \frac{(1+2 \Delta)}{(1+4 \Delta)}$ (with this value occuring at $\Delta^{\prime}=\frac{\Delta}{1+4 \Delta}$ ). Therefore, the incentive to deviate in any $(p, q)$ that is $\Delta$-far from $\left(p^{*}, q^{*}\right)$ is at least this large. Solving for $\Delta$ as a function of $\epsilon$, this game is $(\epsilon, \Delta)$-approximation stable for $\Delta=\epsilon+O\left(\epsilon^{2}\right)$.

Game 3: Rock, Paper, Scissors.

$$
R=\left[\begin{array}{ccc}
0.5 & 0 & 1 \\
1 & 0.5 & 0 \\
0 & 1 & 0.5
\end{array}\right] \quad C=\left[\begin{array}{ccc}
0.5 & 1 & 0 \\
0 & 0.5 & 1 \\
1 & 0 & 0.5
\end{array}\right]
$$

A case analysis (omitted) shows that this game is $(\epsilon, \Delta)$-approximation stable for $\Delta=$ $4 \epsilon$, for any $\epsilon \leq \frac{1}{6}$.

\section{The Support of Equilibria in Stable Games}

We now show that approximation-stable games have structure that can be used to improve the efficiency of algorithms for computing approximate equilibria.

Theorem 1. For any game satisfying $(\epsilon, \Delta)$-approximation stability, there exists an $\epsilon$ equilibrium where each player's strategy has support $O\left((\Delta / \epsilon)^{2} \log (1+1 / \Delta) \log n\right)$.

Corollary 2. There is an algorithm to find $\epsilon$-equilibria in games satisfying $(\epsilon, \Delta)$ approximation stability, running in time $n^{O\left((\Delta / \epsilon)^{2} \log (1+1 / \Delta) \log n\right)}$.

Let $S=c(\Delta / \epsilon)^{2} \log n$ for some absolute constant $c$, and let $\left(p^{*}, q^{*}\right)$ denote the Nash equilibrium such that all $\epsilon$-equilibria lie within distance $\Delta$ of $\left(p^{*}, q^{*}\right)$. Theorem 1 is proven in stages. First, in Lemma 1 we show that given a pair of distributions $(p, q)$, if $p$ is near-uniform over a large support then $p$ can be written as a convex combination $p=x p_{1}+(1-x) p_{2}$ where $p_{1}$ and $p_{2}$ have disjoint supports, and for every column $j$, $j$ 's performance against $p_{1}$ is close to its performance against $p_{2}$. This implies $p^{*}$ itself cannot be near-uniform over a large sized support, since otherwise we could write it in this way and then shift $\Delta$ probability mass from $p_{2}$ to $p_{1}$, producing a new distribution $p^{\prime}$ such that under $\left(p^{\prime}, q^{*}\right)$, the column player has less than $\epsilon$ incentive to deviate (and the row player has zero incentive to deviate since $\left.\operatorname{supp}\left(p^{\prime}\right) \subseteq \operatorname{supp}\left(p^{*}\right)\right)$. This contradicts the fact that the game is $(\epsilon, \Delta)$-approximation stable. We then build on this to show that if $p^{*}$ is not near-uniform and does have a large support, it must be well-approximated by a distribution of small support (roughly $O\left(S \log \frac{1}{A}\right)$ ). This analysis combines Lemma 1 together with the sampling idea of Lipton et al. [11]. The same, of course, applies to $q^{*}$. For the rest of this section we assume that $\Delta \leq 1 / 4$. 
Lemma 1. For any distributions $p$ and $q$, if p satisfies $\|p\|_{2}^{2} \leq \frac{1}{S}$ where $S=c(\Delta / \epsilon)^{2} \log n$ for sufficiently large constant $c$, then $p$ can be written as a convex combination $p=$ $x p_{1}+(1-x) p_{2}$ of two distributions $p_{1}$ and $p_{2}$ over disjoint supports such that:

(i) $x \leq 3 / 4 \leq 1-\Delta$.

(ii) $\forall \bar{j},\left(p_{1}-p\right)^{T} C\left(e_{j}-q\right)<\frac{\epsilon}{4 \Delta}$

The point of Lemma 1 is that by (i) and (ii), modifying $p$ by moving $\Delta$ probability mass from $p_{2}$ to $p_{1}$ can improve the performance of $e_{j}$ relative to $q$ for the column player by at most $\epsilon$. The proof of Lemma 1 makes extensive use of the Hoeffding Bound:

Theorem 2 (Hoeffding Bound). Let $X_{i}, i=1,2, \ldots, n$, be $n$ random variables, s.t. $\forall i, X_{i} \in\left[a_{i}, b_{i}\right]$. Let $\mu_{i}=\mathbf{E}\left[X_{i}\right]$. Then for every $t>0$ we have that:

$$
\operatorname{Pr}\left[\sum_{i} X_{i}>t+\sum_{i} \mu_{i}\right] \leq \exp \left(-\frac{t^{2}}{\sum_{i}\left(b_{i}-a_{i}\right)^{2}}\right)
$$

Proof (Lemma 1). Let $r$ be a random subset of the support of $p$; that is, for every element in $\operatorname{supp}(p)$, add it to $r$ with probability $1 / 2$. Also, let $C_{i}$ denote the $i$ th entry of $C q$. The idea of the proof is just to argue that for any column $j$, by the Hoeffding bound, with high probability over the choice of $r$, the distribution $p_{1}$ induced by $p$ restricted to $r$ satisfies the desired condition that $p_{1}^{T} C\left(e_{j}-q\right)$ is within $\frac{\epsilon}{4 \Delta}$ of $p^{T} C\left(e_{j}-q\right)$. We then simply perform a union bound over $j$.

Fix column $e_{j}$. Let $Y_{i j}$ be the random variable defined as $2 p_{i}\left(C_{i j}-C_{i}\right)$ if element $i$ was added to $r$, and 0 otherwise. Observe that $\mathbf{E}\left[\sum_{i} Y_{i j}\right]=\frac{1}{2} \sum_{i} 2 p_{i}\left(C_{i j}-C_{i}\right)=$ $p^{T} C\left(e_{j}-q\right)$. Let $Z_{i}$ be the random variable defined as $2 p_{i}$ with probability $1 / 2$ (if element $i$ was added to $r$ ), and 0 otherwise. Observe $\mathbf{E}\left[\sum_{i} Z_{i}\right]=1$. Observe also that for every $i$ we have that $Z_{i}, Y_{i j} \in\left[-2 p_{i}, 2 p_{i}\right]$.

The obvious reason for defining $Y_{i j}$ and $Z_{i}$ is that by denoting the distribution $p$ restricted to $r$ (renormalized to have $L_{1}$ norm equal to 1 ) as $p_{r}$, we have:

$$
p_{r}{ }^{T} C\left(e_{j}-q\right)=\frac{\sum_{i \in r} p_{i}\left(C_{i j}-C_{i}\right)}{\sum_{i \in r} p_{i}}=\frac{\sum_{i} Y_{i j}}{\sum_{i} Z_{i}}
$$

so by bounding the numerator from above and the denominator from below, we can hope to find $r$ for which $p_{r}{ }^{T} C\left(e_{j}-q\right)<\mathbf{E}\left[\sum_{i} Y_{i j}\right]+(\epsilon / 4 \Delta)$, thus decomposing $p$ into the desired $p_{1}=p_{r}$ and $p_{2}=p_{\bar{r}}$. We can do this using the Hoeffding bound and plugging the value of $S$ :

$$
\operatorname{Pr}\left[\sum_{i} Y_{i j}>p^{T} C\left(e_{j}-q\right)+\frac{\epsilon}{10 \Delta}\right]<\exp \left(\frac{-(\epsilon / 10 \Delta)^{2}}{\sum_{i}\left(4 p_{i}\right)^{2}}\right) \leq \exp \left(\frac{-S \epsilon^{2}}{(40 \Delta)^{2}}\right)<\frac{1}{2 n},
$$

where the last inequality is by definition of $S$. Thus, $\operatorname{Pr}\left[\exists j, \sum_{i} Y_{i j}>p^{T} C\left(e_{j}-q\right)+\right.$ $\left.\frac{\epsilon}{10 \Delta}\right]<1 / 2$. Similarly (and even simpler), we have that $\operatorname{Pr}\left[\sum_{i} Z_{i}<1-\frac{\epsilon}{10 \Delta}\right]<1 / 2$, and so the existence of $r$ for which both events do not hold is proven. Observe that for this specific $r$ we have that

$$
\frac{\sum_{i} Y_{i j}}{\sum_{i} Z_{i}} \leq \frac{p^{T} C\left(e_{j}-q\right)+\epsilon / 10 \Delta}{1-\epsilon / 10 \Delta} \leq p^{T} C\left(e_{j}-q\right)+\frac{\epsilon / 5 \Delta}{1-\epsilon / 10 \Delta} \leq p^{T} C\left(e_{j}-q\right)+\frac{\epsilon}{4 \Delta},
$$

using the fact that $p^{T} C\left(e_{j}-q\right) \leq 1$. Thus, we have the desired decomposition of $p$. 
Proof (Theorem 1). We begin by partitioning $p^{*}$ into its heavy and light parts. Specifically, greedily remove the largest entries of $p^{*}$ and place them into a set $H$ (the heavy elements) until either (a) $\operatorname{Pr}[H] \geq 1-4 \Delta$, or (b) the remaining entries $L$ (the light elements) satisfy the condition that $\forall i \in L, \operatorname{Pr}[i] \leq \frac{1}{S} \operatorname{Pr}[L]$ for $S$ as in Lemma 1, whichever comes first. We analyze each case in turn.

If case (a) occurs first, then clearly $H$ has at most $S \log (1 / 4 \Delta)$ elements. We now simply apply the sampling argument of Lipton et al [11] to $L$ and union the result with $H$. Specifically, decompose $p^{*}$ as $p^{*}=\beta p_{H}+(1-\beta) p_{L}$, where $\beta$ denotes the total probability mass over $H$. Applying the sampling argument of [11] to $p_{L}$, we have that by sampling a multiset $\mathcal{X}$ of $S$ elements from $\operatorname{supp}\left(p_{L}\right)=L$, we are guaranteed, by definition of $S$, that for any column $e_{j},\left|\left(U_{\mathcal{X}}\right)^{T} C e_{j}-p_{L}^{T} C e_{j}\right| \leq(\epsilon / 8 \Delta)$, where $U_{\mathcal{X}}$ is the uniform distribution over $\mathcal{X}$. This means that for $\tilde{p}=\beta p_{H}+(1-\beta) U_{\mathcal{X}}$, all columns $e_{j}$ satisfy $\left|p^{* T} C e_{j}-\tilde{p}^{T} C e_{j}\right| \leq \epsilon / 2$. We have thus found (the row portion of) an $\epsilon$-equilibrium with support of size $S(1+\log (1 / 4 \Delta))$ as desired, and now simply apply the same argument to $q^{*}$.

If (b) occurs first, we show that the game cannot satisfy $(\epsilon, \Delta)$-approximation stability. Specifically, let $p_{L}$ denote the induced distribution produced by restricting $p^{*}$ to $L$ and renormalizing so that $\sum_{i}\left(p_{L}\right)_{i}=1$, then $\sum_{i}\left(p_{L}\right)_{i}^{2} \leq \frac{1}{S} \sum_{i}\left(p_{L}\right)_{i}=\frac{1}{S}$. Using Lemma 1, we deduce we can write $p_{L}$ as a convex combination $p_{L}=x p_{1}+(1-x) p_{2}$ of $p_{1}$ and $p_{2}$ satisfying the properties of Lemma 1. Again, by denoting $\beta$ as the total probability mass over $H$, we have:

$$
p^{*}=\beta p_{H}+(1-\beta) x p_{1}+(1-\beta)(1-x) p_{2}
$$

where $p_{H}$ is the induced distribution over $H$. We now consider the transition from $p^{*}$ to $p^{\prime}$ defined as

$$
p^{\prime}=\beta p_{H}+((1-\beta) x+\Delta) p_{1}+((1-\beta)(1-x)-\Delta) p_{2}
$$

Notice that by Lemma $1, x \leq \frac{3}{4}$ and hence $(1-\beta)(1-x)-\Delta \geq(1-\beta) / 4-\Delta \geq 0$, so $p^{\prime}$ is a valid probability distribution. Also, since $p_{1}$ and $p_{2}$ are distributions over disjoint support, $p^{\prime}$ is $\Delta$ far from $p^{*}$. Note that since $p^{\prime}$ is obtained from an internal deviation within the support of $p^{*}$, the row player has no incentive to deviate when playing $p^{\prime}$ against $q^{*}$. So, if the game is $(\epsilon, \Delta)$-approximation stable, then playing $p^{\prime}$ against $q^{*}$ must cause the column player to have more then $\epsilon$ incentive to deviate. However, by transitioning from $p^{*}$ to $p^{\prime}$ the expected gain of switching from $q^{*}$ to any $e_{j}$ is

$$
\begin{aligned}
p^{T} C\left(e_{j}-q\right) & =\left(p^{*}+\Delta\left(p_{1}-p_{2}\right)\right)^{T} C\left(e_{j}-q^{*}\right) \\
& \leq \Delta\left(p_{1}-p_{2}\right)^{T} C\left(e_{j}-q^{*}\right) \quad\left(\text { since } p^{* T} C q^{*} \geq p^{* T} C e_{j}\right)
\end{aligned}
$$

From Lemma 1 we know that for every column $j,\left(p_{1}-p_{L}\right)^{T} C\left(e_{j}-q^{*}\right)<\frac{\epsilon}{4 \Delta}$. Also we have that $p_{2}=\frac{1}{1-x}\left(p_{L}-x p_{1}\right)$. Using this we can write $\Delta\left(p_{1}-p_{2}\right)^{T} C\left(e_{j}-q^{*}\right)=$ $\frac{\Delta}{1-x}\left(p_{1}-p_{L}\right)^{T} C\left(e_{j}-q^{*}\right)<\frac{\Delta}{1-x}\left(\frac{\epsilon}{4 \Delta}\right) \leq \epsilon$ where the last step follows from $x \leq 3 / 4$. So the column player has less than $\epsilon$ incentive to deviate which contradicts the fact that the game is $(\epsilon, \Delta)$-approximation stable. 


\section{Polynomial-Time Algorithms when $\Delta$ and $\epsilon$ are Close}

We now show that if $\Delta \leq 2 \epsilon-6 \epsilon^{2}$, then there must exist an $O(\epsilon)$-equilibrium where each player's strategy has support $O(1 / \epsilon)$. Thus, in this case, for constant $\epsilon$, we have a polynomial-time algorithm for computing $O(\epsilon)$-equilibria.

Theorem 3. For any game satisfying $(\epsilon, \Delta)$-approximation stability for $\Delta \leq 2 \epsilon-$ $6 \epsilon^{2}$, there exists an $O(\epsilon)$-equilibrium where each player's strategy has support $\bar{O}(1 / \epsilon)$. Thus, $O(\epsilon)$-equilibria can be computed in time $n^{O(1 / \epsilon)}$.

Proof. Let $\left(p^{*}, q^{*}\right)$ be a Nash equilibrium of the game. First, if there is no set $S$ of rows having a combined total probability mass $x \in[\Delta, \Delta+\epsilon]$ in $p^{*}$, then this implies that except for rows of total probability mass less than $\Delta$, all rows in the support of $p^{*}$ have probability greater than $\epsilon$. Therefore, $p^{*}$ is $\Delta$-close to a distribution of support at most $1 / \epsilon$. If this is true for $q^{*}$ as well, then this implies $\left(p^{*}, q^{*}\right)$ is $\Delta$-close to a pair of strategies $(p, q)$ each of support $\leq 1 / \epsilon$, which by Claim 1 and the assumption $\Delta<2 \epsilon$, is an $O(\epsilon)$-equilibrium as desired. So, to prove the theorem, it suffices to show that if such a set $S$ exists, then the game cannot satisfy $(\epsilon, \Delta)$-approximation stability for $\Delta \leq 2 \epsilon-6 \epsilon^{2}$.

Therefore, assume for contradiction that $p^{*}$ can be written as a convex combination

$$
p^{*}=x p_{1}+(1-x) p_{2},
$$

where $p_{1}, p_{2}$ have disjoint supports and $x \in[\Delta, \Delta+\epsilon]$. Let $\gamma=p_{1}^{T} C q^{*}-p_{2}^{T} C q^{*}$ and let $V_{C}=p^{* T} C q^{*}$. We now consider two methods for moving distance $\Delta$ from $p^{*}$ : moving probability from $p_{1}$ to $p_{2}$, and moving probability from $p_{2}$ to $p_{1}$. Let

$$
\begin{aligned}
p^{\prime} & =(x-\Delta) p_{1}+(1-x+\Delta) p_{2} \\
& =\left(1+\frac{\Delta}{1-x}\right) p^{*}-\left(\frac{\Delta}{1-x}\right) p_{1} .
\end{aligned}
$$

Since $p^{\prime}$ has distance $\Delta$ from $p^{*}$ and its support is contained in the support of $p^{*}$, by approximation-stability, there must exist some column $e_{j}$ such that $p^{\prime T} C e_{j} \geq p^{\prime T} C q^{*}+$ $\epsilon$. By (8) we have $p^{T} C q^{*}=V_{C}-\Delta\left(p_{1}-p_{2}\right)^{T} C q^{*}=V_{C}-\Delta \gamma$. By (9) and the fact that $p^{* T} C e_{j} \leq V_{C}$ we have that $p^{\prime T} C e_{j} \leq V_{C}\left(1+\frac{\Delta}{1-x}\right)$. Therefore we have the constraint

$$
V_{C}\left(1+\frac{\Delta}{1-x}\right) \geq V_{C}-\Delta \gamma+\epsilon .
$$

Now, consider moving $\Delta$ probability mass from $p_{2}$ to $p_{1}$. Specifically, let

$$
\begin{aligned}
p^{\prime \prime} & =(x+\Delta) p_{1}+(1-x-\Delta) p_{2} \\
& =\left(1-\frac{\Delta}{1-x}\right) p^{*}+\left(\frac{\Delta}{1-x}\right) p_{1} .
\end{aligned}
$$

Again, there must exist some column $e_{k}$ such that $p^{\prime \prime T} C e_{k} \geq p^{\prime \prime T} C q^{*}+\epsilon$. By (11) we have $p^{\prime \prime T} C q^{*}=V_{C}+\Delta\left(p_{1}-p_{2}\right)^{T} C q^{*}=V_{C}+\Delta \gamma$. By (12) and the fact that $p^{* T} C e_{k} \leq V_{C}$ we have that $p^{\prime \prime T} C e_{k} \leq V_{C}\left(1-\frac{\Delta}{1-x}\right)+\frac{\Delta}{1-x}$. Therefore we have the constraint

$$
V_{C}\left(1-\frac{\Delta}{1-x}\right)+\frac{\Delta}{1-x} \geq V_{C}+\Delta \gamma+\epsilon
$$

From constraint (10) we have $V_{C}\left(\frac{\Delta}{1-x}\right) \geq \epsilon-\Delta \gamma$ and from constraint (13) we have $V_{C}\left(\frac{\Delta}{1-x}\right) \leq \frac{\Delta}{1-x}-\Delta \gamma-\epsilon$. Therefore, $\frac{\Delta}{1-x} \geq 2 \epsilon$, contradicting $\Delta \leq 2 \epsilon-6 \epsilon^{2}$. 


\section{Stable Games of Large Support}

We now give a near-matching lower bound to the results of Section 3, showing that there exist stable games in which the Nash equilibrium and all approximate equilibria have support $\Omega(\log n)$.

Theorem 4. For any $\Delta \leq 1 / 2$, there exist $n$-by- $n$ games satisfying $(\epsilon, \Delta)$-approximation stability for $\epsilon=\Delta^{2} / 32$ such that all $\epsilon$-Nash equilibria have supports of size at least $(1-\Delta) \lg (n)$.

Thus, Theorem 4 implies the following near-matching lower bound to Theorem 1.

Corollary 3. For any $\Delta \leq 1 / 2$ there exists an $(\epsilon, \Delta)$-approximation stable game $\mathcal{G}$ for some $\epsilon>0$ such that all $\epsilon$-equilibria have support $\Omega\left(\frac{\Delta^{4}}{\epsilon^{2}} \log n\right)$.

Proof. The proof builds on a construction in Feder et al. [10] exhibiting a game in which all approximate equilibria have support of size $\Omega(\log n)$. However, the game in [10] does not satisfy stability and so a more involved construction and argument is needed. We now present the construction of the matrix $R$. The game will be constant sum with $C=1-R$. Let $k=\log _{2}(n)$ and let $\alpha=\Delta / 4$. The matrix R looks like:

Where:

$$
\left[\begin{array}{ll}
X & Y \\
Z & W
\end{array}\right]
$$

- $X$ is $k$ by $k$ with all entries equal to 0.5 .

- $W$ is $n-k$ by $n-k$ with all entries equal to 0.5 .

- $Z$ is $n-k$ by $k$ where each row has $(0.5-\alpha) k$ entries equal to 1 and $(0.5+\alpha) k$ entries equal to 0 . Specifically, all $\left(\begin{array}{c}k \\ (0.5-\alpha) k\end{array}\right)$ different such rows appear. We can add multiple copies of these rows if needed to fill out the matrix.

- $Y$ is $k$ by $n-k$ where each column has $(0.5-\alpha) k$ entries equal to 0 and $(0.5+\alpha) k$ entries equal to 1 . Specifically, all $\left(\begin{array}{c}k \\ (0.5-\alpha) k\end{array}\right)$ different such columns appear. We can add multiple copies of these columns if needed to fill out the matrix.

We begin with two observations about the above construction:

Observation 1: This game has a Nash equilibrium $\left(p^{*}, q^{*}\right)$ which is uniform over the first $k$ rows and columns.

Observation 2: The minimax value of this game is $1 / 2$ to each player. So any $(p, q)$ in which one player gets less than $1 / 2-\epsilon$ is not $\epsilon$-Nash.

We now prove that this game satisfies $(\epsilon, \Delta)$ approximation-stability for $\epsilon=\Delta^{2} / 32$. Let $(p, q)$ be some pair of distributions such that $d\left((p, q),\left(p^{*}, q^{*}\right)\right)>\Delta$. Recall that $d\left((p, q),\left(p^{*}, q^{*}\right)\right)=\max \left[d\left(p, p^{*}\right), d\left(q, q^{*}\right)\right]$ and assume without loss of generality that $d\left(q, q^{*}\right)>\Delta$. We want to show that this is not an $\epsilon$-Nash equilibrium. It will be convenient to write $q=q^{\prime}+q^{\prime \prime}$ where $q^{\prime}$ is nonzero only over the first $k$ columns and $q^{\prime \prime}$ is nonzero only over the remaining $n-k$ columns.

Case 1: Suppose that $\left|q^{\prime \prime}\right|>\beta$ for $\beta=\Delta / 4$. Then, one possible response of the row player is to play $p^{*}$, achieving a payoff $p^{* T} R q$ greater than:

$$
0.5(1-\beta)+(0.5+\alpha) \beta=0.5+\alpha \beta \text {. }
$$


Thus, if $p^{T} R q \leq 0.5+\frac{\alpha \beta}{2}$ then this is not an $\frac{\alpha \beta}{2}$-equilibrium (since the row player would have more than $\frac{\alpha \beta}{2}$ incentive to deviate to $p^{*}$ ) and if $p^{T} R q>0.5+\frac{\alpha \beta}{2}$ then this is also not an $\frac{\alpha \beta}{2}$-equilibrium (since $p^{T} C q=1-p^{T} R q<0.5-\frac{\alpha \beta}{2}$ and yet $p^{T} C q^{*} \geq 0.5$ by Observation 2, so now the column player has more than $\frac{\alpha \beta}{2}$ incentive to deviate). Plugging in $\alpha=\beta=\Delta / 4$, we get $\epsilon=\alpha \beta / 2=\Delta^{2} / 32$ as desired.

Case 2: $\left|q^{\prime \prime}\right| \leq \beta$. Define $d^{\prime}\left(q, q^{*}\right)=\sum_{i=1}^{k} \max \left(q_{i}-q_{i}^{*}, 0\right)$. So, $d^{\prime}\left(q, q^{*}\right)>\Delta-\beta$. For conceptual convenience, let us sort the entries of $q^{\prime}$ (i.e., the first $k$ entries of $q$ ) in decreasing order. We now claim that

$$
\sum_{i=1}^{(0.5-\alpha) k} q_{i}>1 / 2+\alpha \beta
$$

This will imply at least one player has more than $\epsilon$ incentive to deviate since one possible response of the row player is to play the row in matrix $Z$ with 1's in the first $(0.5-\alpha) k$ entries, gaining a value greater than $1 / 2+\alpha \beta$. Thus, if $p^{T} R q \leq 0.5+\alpha \beta / 2$ then the row-player has more than $\alpha \beta / 2$ incentive to deviate to that row in $Z$, and if $p^{T} R q>0.5+\alpha \beta / 2$ then the column player has more than $\alpha \beta / 2$ incentive to deviate to $\left.q^{*}\right)$. So, all that remains is to prove inequality (15). Let $c=q_{(0.5-\alpha) k}$.

Case 2a: $c \geq 1 / k$. In this case we simply use the fact that since the columns are sorted in decreasing order of $q_{i}$, at least an $(0.5-\alpha)$ fraction of the quantity $d^{\prime}\left(q, q^{*}\right)=\sum_{i=1}^{k} \max \left(q_{i}-q_{i}^{*}, 0\right)$ (think of this as the "excess" of $q^{\prime}$ over $\left.q^{*}\right)$ must be in the first $(0.5-\alpha) k$ columns. In addition, we have the remaining "non-excess" $\sum_{i=1}^{(0.5-\alpha) k} \min \left(q_{i}, q_{i}^{*}\right)=[(0.5-\alpha) k](1 / k)=0.5-\alpha$. So, summing these two and using $d^{\prime}\left(q, q^{*}\right)>\Delta-\beta$ we get: $\sum_{i=1}^{(0.5-\alpha) k} q_{i}>$ $(0.5-\alpha)(1+\Delta-\beta)=0.5+\alpha \beta+(0.5 \Delta-0.5 \beta-\alpha-\alpha \Delta) \geq 0.5+\alpha \beta$, where the last inequality comes from our choice of $\alpha=\beta=\Delta / 4$ and assumption that $\Delta \leq 1 / 2$.

Case 2b: $c \leq 1 / k$. This implies that all the $d\left(q, q^{*}\right)-\beta$ "excess" of $q^{\prime}$ over $q^{*}$ must be in the first $(0.5-\alpha) k$ columns. In addition, these columns must contain at least a $(0.5-\alpha)$ fraction of the "non-excess" $\sum_{i=1}^{k} \min \left(q_{i}, q_{i}^{*}\right)$. This latter quantity in turn equals $1-d\left(q, q^{*}\right)$, by using the fact $d\left(q, q^{*}\right)=$ $\sum_{i=1}^{k} \max \left[q_{i}^{*}-q_{i}, 0\right]$. Putting this together we have: $\sum_{i=1}^{(0.5-\alpha) k} q_{i}>(\Delta-$ $\beta)+(0.5-\alpha)(1-\Delta)=0.5-\alpha+\alpha \Delta-\beta+\Delta / 2 \geq 0.5+\alpha \Delta$, where the last inequality comes from our choice of $\alpha=\beta=\Delta / 4$.

This completes Case 2 and the proof.

This example can be extended if desired to make the game be non-constant sum and also so that the sum $R+C$ of the two matrices does not have a constant rank.

\section{Inverse Conditions}

In this section we consider an inverse condition to approximation-stability, namely that for some true equilibrium $\left(p^{*}, q^{*}\right)$, all non-approximate equilibria are far from $\left(p^{*}, q^{*}\right)$. In particular, 
Definition 3. A game is $(\epsilon, \Delta)$-smooth if for some equilibrium $\left(p^{*}, q^{*}\right)$, all strategy pairs $(p, q)$ such that $d\left((p, q),\left(p^{*}, q^{*}\right)\right) \leq \Delta$ are $\epsilon$-equilibria.

We now show that games that are $(\epsilon, \Delta)$-smooth for $\Delta$ large compared to $\epsilon$ have the property that good approximate equilibria can be computed efficiently. (Recall by Claim 1 that all games are $(\epsilon, \Delta)$-smooth for $\Delta \leq \epsilon / 3$.)

Theorem 5. There is a polynomial-time algorithm to find an $(\epsilon / \Delta)$-approximate equilibrium in any game that is $(\epsilon, \Delta)$-smooth.

We prove Theorem 5 through a series of claims as follows.

Claim. Let $\mathcal{G}$ be $(\epsilon, \Delta)$-smooth for equilibrium $\left(p^{*}, q^{*}\right)$. Then for every row $i$ we have $e_{i}^{T} R q^{*} \geq p^{* T} R q^{*}-\epsilon / \Delta$.

Proof. Let $V_{R}=p^{* T} R q^{*}$. Since $\left(p^{*}, q^{*}\right)$ is a Nash equilibrium, any row $e_{i} \in \operatorname{supp}\left(p^{*}\right)$ will get an expected payoff of $V_{R}$ against $q^{*}$ as well. Now consider a row $e_{i} \notin \operatorname{supp}\left(p^{*}\right)$. Let $p=(1-\Delta) p^{*}+\Delta e_{i}$ and consider the pair $\left(p, q^{*}\right)$. This pair is $\Delta$-close to $\left(p^{*}, q^{*}\right)$ and hence, by the assumption that the game is $(\epsilon, \Delta)$-smooth, must be an $\epsilon$-equilibrium. This means that $p^{T} R q^{*} \geq V_{R}-\epsilon$. So we get $(1-\Delta) p^{* T} R q^{*}+\Delta e_{i}^{T} R q^{*} \geq V_{R}-\epsilon$, and using the fact that $p^{* T} R q^{*}=V_{R}$, this implies that $e_{i}^{T} R q^{*} \geq V_{R}-\frac{\epsilon}{\Delta}$.

Similarly, we have:

Claim. Let $\mathcal{G}$ be $(\epsilon, \Delta)$-smooth for equilibrium $\left(p^{*}, q^{*}\right)$. Then for every column $j$ we have $p^{* T} C e_{j} \geq p^{* T} C q^{*}-\epsilon / \Delta$.

Using these claims, we can efficiently compute an $\frac{\epsilon}{\Delta}$-approximate equilibrium in smooth games.

Proof (Theorem 5): Solve the following linear program for a pair of strategies $p, q$ and values $V_{R}, V_{C}$ :

$$
\begin{aligned}
e_{i}^{T} R q & \geq V_{R}-\frac{\epsilon}{\Delta}, & \forall i \\
e_{i}^{T} R q & \leq V_{R}, & \forall i \\
p^{T} C e_{j} & \geq V_{C}-\frac{\epsilon}{\Delta}, & \forall j \\
p^{T} C e_{j} & \leq V_{C}, & \forall j
\end{aligned}
$$

From the previous claims we have that $\left(p^{*}, q^{*}, V_{R}=p^{* T} R q^{*}, V_{C}=p^{* T} C q^{*}\right)$ is a feasible solution to the above LP. Also, when playing $(p, q)$, the row and the column players are getting expected payoff at least $V_{R}-\frac{\epsilon}{\Delta}$ and $V_{C}-\frac{\epsilon}{\Delta}$ respectively. Furthermore, by deviating from $p$, the row player can get a payoff of at most $V_{R}$ and by deviating from $q$, the column player cannot get more than $V_{C}$. Hence, $(p, q)$ is an $\frac{\epsilon}{\Delta}$ approximate Nash equilibrium. 


\section{Open Questions and Conclusions}

In this work we define and analyze a natural notion of approximation-stability for 2player general-sum games, motivated by the goal of finding approximate equilibria for predictive purposes. We show that one can improve over the general Lipton et al. [11] bound based on the extent to which the given game satisfies this condition. Furthermore, if $\Delta<2 \epsilon-O\left(\epsilon^{2}\right)$ we show there must exist approximate equilibria of small support, yielding an algorithm to find them in time $n^{O(1 / \epsilon)}$. On the other hand, we show that approximation-stable games with $\Delta=O(\sqrt{\epsilon})$ can have all approximate equilibria of support $\Omega(\log n)$. We also analyze an inverse condition for which we show finding $(\epsilon / \Delta)$-approximate equilibria can be done efficiently. One open problem is to better understand for what values of $\Delta$ (as a function of $\epsilon$ ) one can find $O(\epsilon)$-approximate equilibria efficiently under the assumption of $(\epsilon, \Delta)$-approximation-stability. For instance, can one extend the $n^{O(1 / \epsilon)}$-time algorithm from $\Delta<2 \epsilon-O\left(\epsilon^{2}\right)$ to $\Delta=\operatorname{poly}(\epsilon)$ ? Recently Balcan and Braverman [3] have shown this may be intrinsically hard: specifically, for $\Delta=\epsilon^{1 / 4}$, they show an $n^{\text {poly(1/ } \epsilon)}$ algorithm to find $\epsilon$-equilibria in such games would imply a PTAS in general games. In fact, [3] motivates the following interesting question: could there be an algorithm that for every $(\epsilon, \Delta)$ finds a $\Delta$-equilibrium in time $O\left(n^{p o l y(1 / \epsilon)}\right)$ ? This may be solvable even if a PTAS is hard for general games, which itself still remains an open question.

\section{References}

1. Timothy G. Abbott, Daniel M. Kane, and Paul Valiant. On the complexity of two-player win-lose games. In Proc. 46th FOCS, pages 113-122, 2005.

2. Maria-Florina Balcan, Avrim Blum, and Anupam Gupta. Approximate clustering without the approximation. In Proc. 20th SODA, pages 1068-1077, 2009.

3. Maria-Florina Balcan and Mark Braverman. Approximate Nash equilibria under stability conditions. Manuscript, 2010.

4. Imre Bárány, Santosh Vempala, and Adrian Vetta. Nash equilibria in random games. Random Struct. Algorithms, 31(4):391-405, 2007.

5. H. Bosse, J. Byrka, and E. Markakis. New algorithms for approximate Nash equilibria in bimatrix games. Theor. Comput. Sci., 411(1):164-173, 2010.

6. Xi Chen and Xiaotie Deng. Settling the complexity of two-player Nash equilibrium. In Proc. 47th FOCS, pages 261-272, 2006.

7. Constantinos Daskalakis, Paul W. Goldberg, and Christos H. Papadimitriou. The complexity of computing a Nash equilibrium. SIAM J. Comput., 39(1):195-259, 2009.

8. Constantinos Daskalakis, Aranyak Mehta, and Christos H. Papadimitriou. Progress in approximate Nash equilibria. In Proc. 8th ACM-EC, 2007.

9. Kousha Etessami and Mihalis Yannakakis. On the complexity of Nash equilibria and other fixed points. SIAM J. Comput., 39(6):2531-2597, 2010.

10. Tomas Feder, Hamid Nazerzadeh, and Amin Saberi. Approximating nash equilibria using small-support strategies. In Proc. 8th ACM-EC, pages 352-354, 2007.

11. Richard J. Lipton, Evangelos Markakis, and Aranyak Mehta. Playing large games using simple strategies. In Proc. 4th ACM-EC, pages 36-41, 2003.

12. H. Tsaknakis and P.G. Spirakis. An optimization approach for approximate Nash equilibria. In WINE, pages 42-56, 2007. 